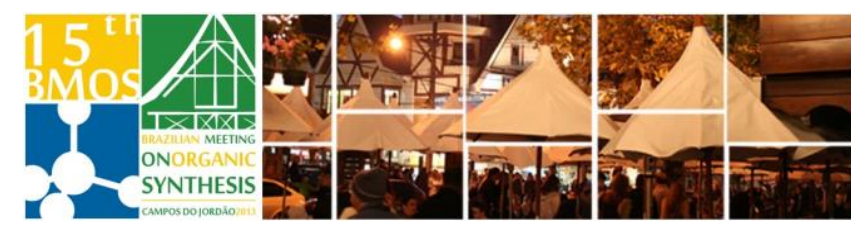

\title{
Synthesis of new resorcinarene-based molecular clips
}

\author{
Miroslav Kvasnica $^{\mathrm{a} \star}$, Byron W. Purse ${ }^{\mathrm{b}}$ \\ ${ }^{a}$ IOCB AS CR, Flemingovo nam. 2, 16610 Praha 6, Czech Republic; ${ }^{b}$ Department of Chemistry and
}

Biochemistry, University of Denver, 2199 S. University Blvd., Denver, Colorado 80208, USA

*e-mail corresponding author: kvasnica@uochb.cas.cz

Keywords: cavitand, molecular clips, resorcinarene

\section{INTRODUCTION}

Molecular clips or tweezers are curved compounds with properties of self-assembly and host-guest recognition. ${ }^{1}$ These clips are capable of binding guests using various supramolecular interactions. They are designed with two 'arms' chosen for their inclination to "sandwich" a given guest. Most examples of resorcinarene-derived cavitand synthesis are symmetrical: the resorcinarenederived deep cavitands either belong to the point groups $\mathrm{C}_{4}$ or $\mathrm{C}_{4 \mathrm{v}}$ (four identical walls) or have one of their walls differentiated by a more elaborate synthesis $\left(\mathrm{C}_{\mathrm{s}}\right.$ symmetry). To date there exists no efficient synthetic methods to control the placement of two different cavitand walls $A$ and $B$ to generate all five possible combinations- $A_{4}, A_{3} B$, cis $A_{2} B_{2}$, trans $A_{2} B_{2}, A_{3}$, and $B_{4}$-because the problem of synthesizing cis $\mathrm{A}_{2} \mathrm{~B}_{2}$ and trans $\mathrm{A}_{2} \mathrm{~B}_{2}$ in more than trace amounts has not been solved. ${ }^{2,3}$ We now present a new regioselective synthesis of the cis $A_{2} B_{2}$ isomer, which will complete the solution to this problem, and in so doing provide a new class of molecular clip or tweezer that is effectively a twowalled deep cavitand.

\section{RESULTS AND DISCUSSION}

Synthesis of resorcinarene cavitand is based mostly on two main sequential steps - preparation of resorcin[4]arene and bridging of phenols with (het)aryls (e.g. 1,2-difluro-4,5-dinitrobenzene DFDNB). Deeper cavitands can be prepared by incorporation of additional walls on upper rim. Cis $\mathrm{A}_{2} \mathrm{~B}_{2}$ cavitands cannot be prepared in high amount by such bridging reactions. If only two equivalents of aryl reagents is used mostly the trans bis-substituted isomers are formed with high amount of trisubstituted and tetra-substituted products. However possible way was found using procedure published by Dalcanale's group ${ }^{4}$. Partial hydrolysis of tetraphosphonate of resorcinarene led to high amount of cis bis-substituted isomer. Free phenolic groups were then easily bridged with DFDNB followed with spontaneous hydrolysis of remaining phosphonates. Reduction of nitro groups to amines and their reactions with benzyl amidate or propionyl chloride led to two precursor for cis $\mathrm{A}_{2} \mathrm{~B}_{2}$ cavitand
(Fig. 1). These compounds, that can be used as molecular clips for specific guests, were further substituted to form cis $\mathrm{A}_{2} \mathrm{~B}_{2}$ cavitands.

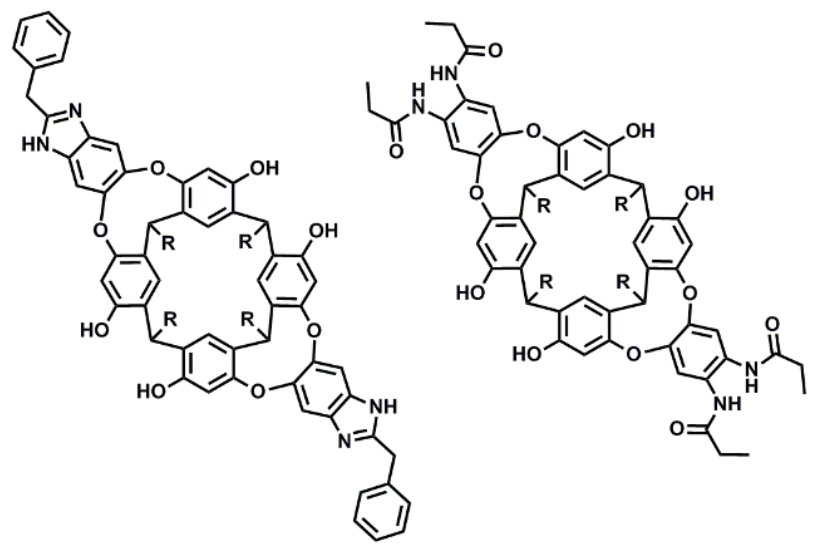

Figure 1. Structure of two molecular clips and precursors for cis $A_{2} B_{2}$ cavitand ( $R=$ alkyl chain).

\section{CONCLUSION}

Our current research on methods for the synthesis of cavitands of reduced symmetry has now provided a new avenue to prepare resorcin[4]arene-based molecular clips and cis $\mathrm{A}_{2} \mathrm{~B}_{2}$ deeper cavitand.

\section{ACKNOWLEDGEMENTS}

Funding for this research was provided by University of Denver and by Czech Ministry of Education, Youth and Sports (Research Project RVO 61388963).

\section{REFERENCES}

${ }^{1}$ Hardouin-Lerouge, M.; Hudhomme, P.; Salle, M. Chem. Soc. Rev., 2011 $40,30$.

${ }^{2}$ Castro, P. P.; Zhao, G.; Masangkay, G. A.; Hernandez, C.; GutierrezTunstad, L. M. Org. Let., 2004, 6, 333.

${ }^{3}$ Vachon, J.; Harthong, S.; Dubessy, B.; Dutasta, J.-P.; Vanthuyne, N.;

Roussel, C.; Naubron, J.-V. Tetrahedron: Asymmetry, 2010, 21, 1534.

${ }^{4}$ Cantadori, B.; Betti, P.; Boccini, F.; Massera, C.; Dalcanale, E. Supramol. Chem., 2008, 20, 29 